\title{
Narrativa
}

\section{Mythos contra Logos. Sulla costruzione e decostruzione del mito del guerriero in Insciallah di Oriana Fallaci}

\section{Barbara Kornacka}

\section{OpenEdition}

\section{Journals}

\section{Edizione digitale}

URL: https://journals.openedition.org/narrativa/539

DOI: $10.4000 /$ narrativa. 539

ISSN: 2804-1224

\section{Editore}

Presses universitaires de Paris Nanterre

\section{Edizione cartacea}

Data di pubblicazione: 1 décembre 2018

Paginazione: 93-103

ISBN: 978-2-84016-325-1

ISSN: $1166-3243$

Notizia bibliografica digitale

Barbara Kornacka, «Mythos contra Logos. Sulla costruzione e decostruzione del mito del guerriero in Insciallah di Oriana Fallaci», Narrativa [Online], 40 | 2018, online dal 01 novembre 2021, consultato il 08 décembre 2021. URL: http://journals.openedition.org/narrativa/539 ; DOI: https://doi.org/10.4000/ narrativa.539 


\title{
Mythos contra Logos. \\ Sulla costruzione e decostruzione del mito del guerriero in Insciallah di Oriana Fallaci
}

\begin{abstract}
RIASSUNTO
L'articolo si concentra su due procedimenti opposti presenti nel romanzo Insciallah di Fallaci: la creazione del mito del guerriero e la sua decostruzione grazie alla forza del logos. La costruzione del mito del guerriero si basa sull'ideologia della mascolinità, sull'elogio della guerra e del mestiere di soldato e si convalida con i modelli prodotti dalla cultura. Nel processo della decostruzione del mito l'autrice mette in discussione la professione di soldato, denuda l'assurdità della guerra nonché deride il simbolo del potere maschile - il fallo - destituendo così di fondamento il connubio tra la mascolinità e il mito del guerriero.

\section{RÉSUMÉ}

L'article se concentre sur deux mécanismes opposés que l'on retrouve dans le roman Insciallah de Fallaci : la création du mythe du guerrier et sa déconstruction grâce à la force du logos. La construction du mythe du guerrier est basée sur l'idéologie de la masculinité, sur l'éloge de la guerre et du métier du soldat; elle est par la suite validée par les modèles produits par la culture. Le processus de déconstruction met en cause la profession du soldat, révèle l'absurdité de la guerre et discrédite le symbole du pouvoir masculin - le phallus - révoquant l'union entre masculinité et mythe du guerrier.
\end{abstract}

Tnsciallah, romanzo di Oriana Fallaci uscito in Italia nel luglio del $1990^{1}$, è un mondo a sé stante: David M. Turoldo scrive che anticiparne il contenuto sarebbe "come riassumere la storia del mondo". È un libro immenso che

1. Edizione di riferimento, da cui sono tratte le citazioni: FALLACI Oriana, Insciallah, Milano, Rizzoli, 2016.

2. Turoldo M. Davide, "Viaggio nel vulcano Insciallah", Corriere della Sera, 2 agosto 1990. 
offre numerosi spunti di riflessione, intrecciandovi l'autrice le sue osservazioni sul Fato, sul Destino, sul Caos, sulla Vita, sulla Morte, e innanzitutto sugli Uomini, che "sanno costruire le strade e i ponti e le case, sanno dipingere la Cappella Sistina e scrivere l'Amleto e comporre il Nabucco e trapiantare il cuore e andare sulla Luna ma sono peggiori delle bestie"3.

Tra i temi e motivi dell'opera vi è il soldato ${ }^{4}$, ovvero il moderno guerriero. Nelle pagine che seguono si propone un'analisi della costruzione e della decostruzione del mito del guerriero, procedimenti opposti che, all'avviso di chi scrive, si intrecciano, si susseguono e si mettono continuamente in atto nel testo della Fallaci. In altre parole, si tenderà a ricostruire il complesso gioco in cui l'autrice palesa il meccanismo della costruzione del mythos - in questo caso della mitica, arcaica e contemporanea, figura del guerriero - smantellato e contrariato dal logos, identificato con la ragione, il pensiero, la parola e opposto all'ordine del mythos ${ }^{5}$.

\section{MYTHOS}

Prima tuttavia di procedere con un'analisi impostata sul contrasto tra mythos e logos e incentrata sul mito del guerriero nell'opera fallaciana, si deve dedicare una breve riflessione al mito stesso, volendo precisare l'accezione data alla parola in questa sede. Secondo la spiegazione fornitaci dal dizionario dei termini etnografici, il mito, in primo luogo, può essere inteso come un racconto sulle divinità, sull'origine dell'universo, dell'essere umano e dei fenomeni. In secondo luogo va inteso come un'arcaica visione del mondo, espressa attraverso la simbologia che si riferisce alla natura e all'organizzazione dell'universo. Infine, il mito è una forma di coscienza universale e comune entro la quale vengono associati, in maniera simbolica, alcuni fenomeni e alcune $\operatorname{cose}^{6}$. Sarà quest'ultima

3. Fallaci Oriana, Insciallah, cit., p. 519.

4. Vi sono più di una trentina di soldati, protagonisti del romanzo, una collettività $e$ al contempo ognuno con una storia individuale.

5. BurszTA Wojciech Józef, "Logos i mythos w antropologii kultury”, in WysŁouch Seweryna, Kaniewska Bogumiła et al. (a cura di), Logos i mythos w kulturze XX wieku, Poznań, Wydawnictwo Poznańskie Studia Polonistyczne, 2003, p. 23. L’opposizione tra logos e mythos non riguarda tuttavia la cultura antica greca, dove il mito era in simbiosi con la sfera del logos diventando un importante elemento della razionalità di quel periodo. Cfr. TrzciŃsKA Izabela, Logos, mit i ratio: wybrane koncepcje racjonalności od XV do XVII wieku, Kraków, Wydawnictwo Uniwersytetu Jagiellońskiego, 2011, p. 40.

6. Tomicki Ryszard, voce "Mit", in Staszczak Zofia (a cura di) Słownik etnologiczny. Terminy ogólne, Warszawa, PIW, 1987, pp. 244-248. 
accezione di mythos cui si riferiranno le considerazioni e le analisi che seguono: il mito come una determinata forma di coscienza "più profonda" che possiede la sua funzione sociale precisa e atemporale, quella di integrare un gruppo attraverso un processo che si svolge senza la partecipazione della coscienza "razionale", quella di unire le persone, creando al contempo un legame con la realtà esterna ${ }^{7}$. Inteso in questo modo, il mito si avvicina all'archetipo, all'immagine collettiva simbolica, veicolo di credenze, convinzioni ed emozioni comuni per un insieme di persone. Inoltre, si ritiene valido in questa sede quanto sostenuto Joseph Campbell secondo cui i miti al piano di esistenza e quello strutturale offrono modelli di vita che vanno modificati e adeguati all'epoca in cui si vive, agli ideali e valori validi in quel tempo ${ }^{8}$.

\section{CostruZione DEL MITO DEL GUERRIERO}

Le relazioni tra letteratura e mito sono multiple, bilaterali e bidirezionali. Da un lato nelle opere letterarie, dall'epos al fumetto, "trapelano" - talvolta anche senza un consapevole controllo da parte dell'autore - elementi, motivi o trasposizioni dei miti'; dall'altro, nelle varie epoche storiche i testi letterari possono generare miti ${ }^{10}$. La mitica figura del guerriero, emblema di virilità, coraggio, audacia, eroismo e forza, è infatti meglio illustrata (e in parte perfino generata) nell'Iliade che ne diffonde l'immagine di secolare durabilità. Come sostiene Tomasz Tomasik questo epos divenne veicolo letterario del nobilitante ethos di uomo come eroe e guerriero ${ }^{11}$. Oriana Fallaci, ricorrendo alla strategia dell'alter ego romanzesco attraverso il personaggio del Professore, che nel gioco metaletterario confessa di voler scrivere un romanzo ispirato agli avvenimenti e ai personaggi circostanti, definisce Insciallah una piccola Iliade: "una piccola Iliade si muove attorno a me: una moderna Iliade in trentaduesimo, dove con un po' di umorismo si trovano quasi tutti gli eroi del divino poema" ${ }^{\prime 2}$. Elencando in

7. Cassirer Ernest, The individual and the Cosmos in Renaissance Philosophy, Oxford, Basil Blackwell, 1963, pp. 120-130, cit. da BurszTA Wojciech Józef, "Logos I mythos w antropologii kultury", cit., p. 24.

8. Campbell Joseph, The Power of Myth, New York, Doubleday,1988.

9. Łotman Jurij, Minz Zara, "Literatura i mitologia", Pamiętnik Literacki, 82/1, 1991, p. 242.

10. Ibid, p. 251.

11. Tomasik Tomasz, Wojna - męskość - literatura, Słupsk, Wydawnictwo Naukowe Akademii Pomorskiej w Słupsku, 2013, pp. 58-59.

12. Fallaci Oriana, Insciallah, cit., p. 215. 
seguito le analogie tra personaggi ed episodi di entrambe le opere, l'Iliade e Insciallah, constata: "Paragoni fittizi o no, pretestuosi o no, la storia non cambia. L'eterna storia, l'eterno romanzo dell'Uomo che alla guerra si manifesta in tutta la sua verità"'13. La scrittrice, concependo il suo libro come una piccola Iliade, come un epos guerresco contemporaneo, analogo per più ragioni a quello antico, traccia dunque un parallelismo che collega gli antichi guerrieri e i soldati moderni. Di conseguenza autorizza la lettura della sua opera anche in chiave mitologica: ovvero consente di individuare nel suo romanzo il tema del mito del guerriero, sempre operativo, il quale si attua, in primo luogo, attraverso l'associazione con l'idea di mascolinità, in seguito attraverso l'elogio della guerra e del mestiere di soldato e infine attraverso diversi modelli di guerrieri prodotti dalla cultura e dalla Storia.

La potenza di questo mito si fonda innanzitutto sul suo indissolubile legame con la mascolinità che, come spiega Elisabeth Badinter, al contrario della femminilità, non è vista come una condizione naturale, innata, bensì prodotta, conquistata e provata ${ }^{14}$. Nelle società arcaiche il raggiungimento dello status di maschio avveniva grazie ai riti di iniziazione ${ }^{15}$. In quella contemporanea invece modi e metodi per trovare e confermare la propria identità di uomo sono testati intuitivamente ${ }^{16}$. Si ammette che proprio la guerra sia un rituale di iniziazione in cui i giovani ragazzi sono sottoposti alla prova di mascolinità. La promessa di mascolinità sconfigge la naturale ritrosia dei giovani ragazzi a partecipare alla guerra ${ }^{17}$. In altre parole, essere guerriero comporta in sé la promessa dell'agognata mascolinità.

Questo connubio della mascolinità e del mito del guerriero, Fallaci lo evidenzia più volte nel suo romanzo, esponendo i princìpi sui quali si basa la vitalità e validità di questo mito, in particolar modo per alcuni dei protagonisti. È il caso soprattutto di giovani soldati di basso rango, venuti a Beirut come volontari, nel cui ragionamento si osserva una diretta e genuina associazione tra essere maschi e partecipare alla guerra, la quale "fa bene perché qui si danno gli

13. Ibid., p. 217.

14. Badinter Elisabeth, X Y. De l'identité masculine, Parigi, Odile Jacob, 1992.

15. GenNeP Arnold Van, Les Rites de passage, Ed. Nourry, 1909.

16. Cfr. La Cecla Franco, Modi bruschi. Antropologia del mascbio, Milano, Eleuthera, 2000. Cfr. KornaCKA Barbara, "Quasi adulti? I riti di passaggio nella prosa dei 'giovani narratori' italiani della fine dẹl Novecento", Romanica Silesiana, Rites et cérémonies, n. 9, Wydawnictwo Uniwersytetu Sląskiego, Katowice, 2014, pp. 237-246.

17. TomasiK Tomasz, Wojna - męskość - literatura, cit., p. 28. 
esami di maturità"18. È la guerra a offrire opportunità, "di diventare un uomo"19. Gino che a quindici anni andava a caccia ${ }^{20}$ e "a sparare si sentiva un uomo"21, scegliendo l'esercito parte dal presupposto, tramandatogli dagli antenati, che la violenza e l'aggressione sono attributi della mascolinità. Fallaci tuttavia nella stessa frase aggiunge: "e non glielo spiegava nessuno che meno si spara più uomini si è", destituendo così di fondamento un tale ragionamento. L'idea di equipollenza tra mascolinità e prestazioni belliche che sta alla base della costruzione del mito viene esposta nel romanzo in diversi episodi. Gli atteggiamenti e le convinzioni dei giovani soldati confermano ciò che Fallaci intende con la sua opera denudare e controbattere, in particolare che la guerra sia un'esperienza "naturale" nella quale si forma il "vero" carattere dell'uomo e l'identità maschile. È una convinzione erronea però valida dai tempi più remoti, come sostiene Tomasik, che essere uomini significa partecipare alla guerra, essere guerrieri ${ }^{22}$.

Il mito del guerriero inoltre si costruisce e si rafforza, come dimostra Fallaci, con l'elogio delle qualità del mestiere di soldato nonché dei pregi della guerra stessa da parte di alcuni ufficiali di alto rango, dediti da anni alla carriera militare, tra cui in primis Condor, generale della spedizione italiana a Beirut, nonché, egli stesso, icona del guerriero contemporaneo per eccellenza:

[...] una delle creature che coabitavano in lui aveva la debolezza di piangere. Le altre, invece, si distinguevano per la baldanza, l'albagia, e la capacità di far piangere il prossimo. Le guidava un orgoglio smisurato, un esasperato bisogno di emergere anzi di vincere, la peculiarità del personaggio nasceva in gran parte da quei difetti peraltro alimentati dai doni con cui gli dèi lo avevano favorito: l'intelligenza, il coraggio, la salute di chi non invecchia mai. A 55 anni ne dimostrava appena 40 e sul volto dai lineamenti armoniosi non scorgevi nemmeno una ruga. Il suo corpo era svelto, la sua camminata sciolta, il suo fascino riconosciuto ${ }^{23}$.

18. FallaCi Oriana, Insciallah, cit., p. 104.

19. Ibid., p. 525.

20. Secondo Barbara Ehrenreich la caccia sta all'origine delle prime guerre dell'umanità rivolte contro gli animali, nel momento in cui l'uomo dalla vittima di essi si trasforma, egli stesso, in predatore. Cfr. Ehrenreich Barbara, Blood Rites, York, Metropolitan Books, 1997.

21. Fallaci Oriana, Insciallah, cit., p. 129.

22. Tomasik Tomasz, Wojna-męskość - literatura, cit., p. 24.

23. Fallaci Oriana, Insciallah, cit., p. 65. 
La dimensione mitica del personaggio viene accentuata tra l'altro dal richiamo agli dei che lo corredano con le sue qualità del guerriero. Condor ama la guerra così come la ama anche Zucchero, un altro ufficiale e "un militare nato" che "si compiaceva d'essere una rotella dell'ingranaggio. "Il mio mestiere è il più bello del mondo' asseriva. 'Non lo cambierei nemmeno per diventare re o miliardario"" 24 . Tra i fautori del mestiere di soldato e della guerra stessa, i quali ne cantano la bellezza, "la vitalità (per quanto possa sembrare paradossale) che la guerra porta dentro di sé" 25 , vi sono inoltre Pistoia, Cavallo Pazzo e Sandokan, sempre ufficiali, definiti da Oriana come veri soldati che "amano in modo profondo la guerra" 26 , che è "un grande esame" 27 e "la linfa della vita: vita che nasce con la vita, che scorre nelle vene dell'uomo insieme al suo sangue" 28 . Celandosi dietro questi personaggi l'autrice abilmente ricostruisce l'ideologia militare e la propaganda bellica - suggerendone l'irrazionalità e l'assurdità - di cui essi sono portatori. La stessa ideologia militare e la propaganda bellica, contribuiscono a generare la mitologia guerresca la quale, a sua volta, le nutre e sostiene con immagini e figure create dalla letteratura e dalla cultura di massa.

I diversi modelli di guerrieri, emblemi del mito - che siano letterari, cinematografici o storici - in quanto ideali seguiti da alcuni protagonisti che ne vogliono imitare l'esempio, riaffermano nonché diffondono il mito. Fallaci ne riporta soltanto alcuni, rilevanti per alcuni personaggi come, per Cavallo Pazzo, l'eroico Des Aix, morto nella battaglia di Marengo nel 1800 o Don Chisciotte che "vive nel rimpianto di un passato eroico" ${ }^{\prime 2}$. Per Charlie invece Lawrence d'Arabia era "ciò che Antoine-Charles-Louis Collinet conte di Lasalle e Louis-Charles-Antoine Desaix anzi Des Aix cavaliere di Veygoux erano per Cavallo Pazzo: un modello, un maestro" 30 . Invece Sandokan si formò il suo ideale del guerriero seguendo le pellicole di guerra: il suo mitico guerriero aveva il volto di Henry Fonda, John Wayne o Robert Mitchum che diventano "una fissazione. E mentre la fissazione copriva le pareti della sua camera con fotografie di cacciatorpediniere, motocannoniere, incrociatori, fregate, corvette, posamine, sommergibili, il ragazzo educato nel pacifismo si trasformava sempre di più in un guerraiolo"31.

24. Ibid., p. 123.

25. Ibid., p. 151.

26. Ibid.

27. Ibid., p. 240.

28. Ibid., p. 303.

29. Ibid., p. 337.

30. Ibid., p. 83.

31. Ibid., p. 306. 
La cultura, alta e bassa, nonché la Storia partecipano alla costruzione, alla sopravvivenza e alla resistenza del mito.

\section{LOGOS OVVERO SULLA DECOSTRUZIONE DEL MITO}

Nonostante il carattere persistente e duraturo del mito del guerriero, Fallaci lo scompone con l'arma del logos ovvero dell'argomentazione razionale e logica. È un compito attribuito in maniera corale, oltre che all'autrice stessa (narratore) e all'intelletto in generale (figura del Professore, emblema dello studioso). Inoltre, hanno voce in capitolo alcuni importanti rappresentanti dell'esercito stesso nonché, cosa interessante, gli individui marginalizzati e periferici sia nella struttura romanzesca sia quella sociale (soldato omosessuale e soldati semplici). La decostruzione del mito del guerriero si effettua per mezzo di alcune strategie quali la critica della guerra contrastata dall'elogio della vita, la contestazione della professione di soldato e la messa in ridicolo del culto del fallo, strategie che ora si cercherà di analizzare.

Il tema della guerra è cruciale nel romanzo e l'opinione della Fallaci a questo proposito è uno dei fili conduttori del libro. Oltre alla scontata violenza, crudeltà, bruttezza e morte che emanano dalle immagini della guerra, Fallaci ce la presenta come spazio e tempo governati dal caos, dall'assurdità, dalla stupidità e oltretutto dal caso. Gli esempi sarebbero numerosi ma le più spettacolari e significative pagine sono quelle dedicate alla pallottola 5,56 veloce tre volte la velocità del suono e leggerissima, con la gittata di tre chilometri. Una di queste, avendo mancato il suo bersaglio, raggiunge una tenda di un accampamento e penetra la nuca di Rocco, un soldato qualunque ma con la sua storia d'amore e le sue speranze di vita, mentre si accinge a dormire e a sognare della sua amata Imam, e avendogli girato il cervello lo lascia cerebroleso. Molti soldati, protagonisti del romanzo, odiano la guerra proprio per il caos che produce, per la sua mancanza di senso e per la sua inutilità. Tra questi la voce importante è quella di Charlie

che detestava la guerra più dei pacifisti in borghese. La guerra non serve a nulla, diceva, non risolve nulla. Appena una guerra è finita ti accorgi che i motivi per cui era scoppiata non sono scomparsi, o che se ne sono aggiunti di nuovi in seguito ai quali ne scoppierà un'altra dove gli ex nemici saranno gli amici e gli ex amici i nemici. La guerra è figlia della violenza che a sua volta è figlia della forza fisica, e il trinomio non partorisce che scelleratezze" ${ }^{\prime 32}$.

32. Ibid., p. 84. 
Esprimono l'odio per la guerra i numerosi soldati arruolati per caso, attratti da qualche ingannevole immaginario offerto dal cinema o dalla letteratura, tra cui Luca che, infelice e arrabbiato contro la sua sorte, incolpa Hemingway. Sono rilevanti i suoi ragionamenti in cui non solo contesta la guerra come iniziazione alla mascolinità, convinzione fondamentale per generazioni di uomini, ma anche dissacra i modelli letterari, cruciali per l'istituzione del mito del guerriero:

Ma che maniera di crescere era questa, che maniera di diventare un uomo? Se diventare un uomo significa trasformarsi in una persona stanca e delusa, meglio restar ragazzi per sempre: Peter Pan che giocano nei giardini di Kensington alla ricerca di Never Never Never Land, il Paese Che Non Esiste. Tutta colpa di Hemingway, maedeto Hemingway, delle sue smargiassate sulla virilità e sul coraggio $[\ldots]$. Non si dovrebbe mai prenderli sul serio gli scrittori, mai. [...] Diventare uomini, conoscer la guerra, affrontar la paura e la morte, cazzate del genere. Maedeto, maedeto! ${ }^{33}$

Forse per questo effetto nocivo della letteratura che trapela dalle parole di Luca, il romanzo scritto dal Professore, ovvero la piccola Iliade, non sarà portato a termine ${ }^{34}$. Insciallah - inizialmente concepito come piccola Iliade - non vuole essere, come lo è l'Iliade di Omero, un elogio della guerra, e quindi anche del caos e della morte. Infatti, il filo che torna con insistenza nel romanzo è la ricerca della formula della Vita da parte di Angelo, soldato matematico, da contrapporre alla formula della Morte di Ludwig Boltzmann. Oriana, nonostante le atrocità della guerra, elogia la vita e i suoi più grandi valori: amore, amicizia, carità e solidarietà, che sono forse quell'“altra bellezza" di cui parla Alessandro Baricco nella sua Postilla sulla guerra, postfazione alla sua riscrittura dell'Iliade di Omero:

Per quanto suoni atroce, è necessario ricordarsi che la guerra è un inferno: ma bello. [...] Per questo, oggi, il compito di un vero pacifismo dovrebbe essere non tanto demonizzare all'eccesso la guerra, quanto capire che solo quando saremo capaci di un'altra bellezza potremo fare a meno di quella che la guerra da sempre ci offre ${ }^{35}$.

\section{Ibid., p. 413.}

34. Rinuncia anche all'ambizione di essere scrittore perché egli, come scrive, "non sempre le idee che anticipa e che trasmette sono ottime idee [...] sono ideologie che schiavizzano e impediscono di avere idee", puntando proprio sulla responsabilità dello scrittore per gli effetti nocivi, il male che la letteratura può generare (ibid., p. 813).

35. Baricco Alessandro, "Un'altra bellezza. Postilla sulla guerra", in Omero, Iliade, Milano, Feltrinelli, 2004, pp. 161-162. 
Tuttavia, come spiega il Professore (alter ego di Fallaci) nella sua ultima lettera: "Il discorso da affrontare non è sulla guerra. È sugli uomini che fanno la guerra, sui soldati, sul mestiere più antico più inalterabile più intramontabile che esista dacché esiste la vita. Il mestiere di soldato. Il mestiere che amavo, che rispettavo, che idealizzavo, e che ora ripudio" ${ }^{36}$. La decostruzione del mito del guerriero passa anche attraverso la contestazione del mestiere di soldato, dell'uniforme dentro cui alcuni degli ufficiali non si sentivano a loro agio sin dall'inizio come Aquila Uno che "non ci teneva ad esser scambiato per un prepotente, un guerriero. Li detestava, i guerrieri. E coi guerrieri le uniformi, le armi. Si sentiva ridicolo in uniforme"37. Per altri invece, come per Zucchero, "era l'improvvisa, inaspettata, insospettata scoperta d'aver sciupato la propria vita a venerare un mestiere in cui di colpo non credeva più. È atroce scoprire d'aver sciupato la propria vita a venerare un mestiere in cui non si crede più" 38 . Altri ancora "amanti della guerra" maturano col tempo e soltanto una volta "tuffati dentro l'atroce esperienza d'una battaglia cambiano in modo radicale. Capiscono d'avere amato qualcosa da odiare e disseppellendo la loro vera natura strappano la maschera che avevano scelto"39. L'analisi più approfondita delle cause che portano al rifiuto del mestiere di soldato ce la fornisce il Professore che non crede più nel mestiere di soldato dal momento in cui esso

consiste anche nell'allargare il territorio di tale tribù, aumentarne la potenza, imporne la fede: compito da assolvere rammentando quel che t'hanno insegnato e cioè che chi non vuole cedere il suo territorio o rinunciare alla sua fede è un nemico, che il nemico è una cosa da distruggere, che distruggerlo è diritto e dovere d'ogni soldato, nonché un privilegio concesso dall'impunità che il mestiere di soldato garantisce ${ }^{40}$.

Il soldato, ragiona il Professore, in nome degli dèi, delle religioni, delle ideologie e dei cosiddetti ideali uccide, sodomizza, stupra, compie atti atroci e delitti crudeli per cui alla fine riceve una medaglia d'oro, istruito che ci sono "due modi di giudicare il Bene e il Male: due opposti concetti del Male e del Bene" ${ }^{\text {"41. }}$. Nelle riflessioni del suo alter ego, Fallaci espone tutta la falsità, l'ipocrisia

36. Fallaci Oriana, Insciallah, cit., p. 807.

37. Ibid., p. 93.

38. Ibid., p. 556.

39. Ibid., p. 590.

40. Ibid., p. 808.

41. Ibid., p. 810. 
e il relativismo su cui poggia il mito del guerriero, modello e ideale militare di virtù della mascolinità.

Un altro aspetto dell'ideologia della mascolinità che il mito del guerriero deve sostenere e ratificare ${ }^{42}$, è quello rappresentato simbolicamente dal fallo ossia quello che identifica il maschio con il potere e con la legge universale dell'umanità $^{43}$. È un aspetto che Fallaci mette in discussione, ridicolizzandolo per bocca di Mattia, soldato omosessuale. È questo ragazzo "diverso" a osservare che tra i militari vige un eccessivo culto del fallo, "la glorificazione anzi la deificazione del testicolo, l'esaltazione anzi l'apoteosi del cazzo visto come simbolo di virilità" ${ }^{44}$. Nella sua acuta analisi dimostra che nell'esercito, che non accetta l'omosessualità, paradossalmente, il culto del fallo è pari a quello che vige tra gli omosessuali:

verso il fallo i militari hanno un culto quasi più profondo dei froci. [...] $\mathrm{E}$ la bandiera dei militari quel bizzarro cilindro di carne che a 13 anni credevo servisse a fare pipi e nient'altro. È il loro dio, il Dio Fallo: l'emblema della loro arroganza, della loro petulanza, della loro presunzione, del loro maschilismo. Lo citano ad ogni appiglio. [...] Cazzo qua, cazzo là, cazziatone, incazzare, incazzato, incazzata, cazzata. Oppure coglionata, scoglionato, coglione, coglioni. I coglioni visti quale simbolo di coraggio, virilità-uguale-coraggio, i coglioni dell'antitema che considera il coraggio una virtù esclusivamente maschile. L'uniforme, lo strumento di tale virtù. Un-uomo-deve-fare-il-soldato, per-diventareun-uomo-bisogna-fare-il-soldato. Eccetera. Balle! ${ }^{45}$

Martino, in un solo colpo ironico e canzonatorio, riduce e spodesta il fallo, simbolo venerato e poi scredita il virilismo dei militari che in realtà è il travestimento dell'arroganza, della presunzione e del maschilismo.

\section{Conclusione}

Tra i numerosi temi del romanzo analizzato vi è anche quello del soldato e del suo rapporto con il mito del guerriero, emblema di forza, coraggio e virilità. Fallaci ricostruisce il meccanismo della costruzione di tale mito, valido e vivo per una parte dei soldati, il quale poggia sull'ideologia della mascolinità,

42. Secondo Joseph Campbell (The Power of Myth, cit.), una delle funzioni del mito consiste nel convalidare e giustificare un dato ordine sociale.

43. Cfr. BAdinter Elisabeth, XY, cit.

44. Fallaci Oriana, Insciallah, cit., p. 206.

45. Ibid., pp. 386-387. 
sull'elogio della guerra e delle qualità del mestiere di soldato e che si convalida attraverso la valorizzazione dei modelli forniti dalla cultura alta e bassa e dalla Storia. Al contempo l'autrice, applicandovi tutta la forza della ragione e l'acuta intelligenza che le è propria, decostruisce questo mito e in un percorso alla rovescia lo destituisce di fondamento. Con un'argomentazione rivelatrice vengono evidenziate l'assurdità, la stupidità e la casualità della guerra cui è contrapposto l'elogio della vita e dei suoi valori: solidarietà, amicizia e amore. Si procede con una metodica critica del mestiere di soldato per finire con lo screditamento della potenza del simbolo, di antica data, di virilità e di potere maschili, ovvero del fallo, venerato eccessivamente dai militari, procedendo con ciò alla rottura tra l'ideologia della mascolinità e il mito del guerriero.

Barbara KORNACKA Poznan University 
